\title{
Three-body recombination at large scattering lengths in an ultracold atomic gas
}

\author{
Tino Weber, Jens Herbig, Michael Mark, Hanns-Christoph Nägerl, and Rudolf Grimm \\ Institut für Experimentalphysik, Universität Innsbruck, Technikerstr. 25, A-6020 Innsbruck, Austria
}

(Dated: November 2, 2018)

\begin{abstract}
We study three-body recombination in an optically trapped ultracold gas of cesium atoms with precise magnetic control of the s-wave scattering length $a$. At large positive values of $a$, we measure the dependence of the rate coefficient on $a$ and confirm the theoretically predicted scaling proportional to $a^{4}$. Evidence of recombination heating indicates the formation of very weakly bound molecules in the last bound energy level.
\end{abstract}

Three-body recombination, the process of two particles forming a compound while interacting with a third particle, is of general importance in many-body systems. Understanding three-body processes has for a long time been a challenge in many areas of physics. In dilute ultracold atomic gases, where unique experimental access to interparticle interactions is available, three-body recombination leads to the formation of diatomic molecules. The possibility to trap and cool such molecules and thus to realize ultracold molecular ensembles [1] or atom-molecule mixtures 2] holds great prospects for future research in the field of quantum gases.

The main experimental challenge for measuring threebody recombination is to distinguish three-body losses from two-body losses. In magnetic traps, this is in general difficult, as dipolar relaxation is present as an inherent two-body loss mechanism. In the special case of magnetically trapped ${ }^{87} \mathrm{Rb}$, experiments could nevertheless provide reliable data because of the anomalously weak two-body decay in this species [3, 4]. As a more general approach, optical traps allow to store atoms in the lowest internal state where two-body loss is fully suppressed for energetic reasons 5]. Three-body recombination in bosonic systems with large $s$-wave scattering length $a$ has been explored in experiments utilizing Feshbach resonances in ${ }^{23} \mathrm{Na}$ [6] and ${ }^{85} \mathrm{Rb}$ [7]. The results demonstrate the dramatic enhancement of loss processes near such resonances.

In the case of large positive $a$, theoretical studies [8, 9, 10, 11] predict formation of molecules in a weakly bound $s$ level and deduce scaling of the three-body recombination rate with $a$ to the fourth power. Recombination is discussed in terms of the event rate $\nu_{\text {rec }}=\alpha_{\text {rec }} n^{3}$ per unit volume and time, where $n$ denotes the particle density. In the low-energy limit, the universal scaling law for the characteristic parameter $\alpha_{\text {rec }}$ is given as $\alpha_{\text {rec }}=C \hbar a^{4} / m$ [8], where $m$ is the mass of the atom, and $C$ a dimensionless factor. Predictions for $C$ give values between 0 and $\sim 70[8,9,10,11$, with oscillatory behavior between $C=0$ and $C_{\max }=67.9$ expected for strong variations of $a$ on a Feshbach resonance [1]. Previously available experimental data on three-body recombination for various species roughly agree with the general trend of a universal $a^{4}$ scaling, with evidence for deviations from this scaling law near a Feshbach resonance 6].

In this work, we employ an optical trap to measure three-body recombination in an ultracold thermal gas of ${ }^{133} \mathrm{Cs}$ in the $6^{2} \mathrm{~S}_{1 / 2}, F=3, m_{F}=3$ absolute ground state. In this state, the scattering length varies strongly with an applied magnetic field $B$ through a combination of broad and narrow Feshbach resonances at low field strengths [12, 13]. The values of $a$ as a function of $B$ have been calculated to high precision [14]. This tunability allows us to conduct experiments in a range of precisely controlled large positive values of $a$, where the condition of recombination into a weakly bound $s$ level is well fulfilled. Detailed measurements of the time evolution of both atom number and temperature allow us to quantify both three-body loss and heating.

The three-body loss rate coefficient $L_{3}$ is related to $\alpha_{\text {rec }}$ via $L_{3}=n_{l} \alpha_{\text {rec}}$, where $n_{l}$ denotes the number of atoms lost from the trap per recombination event. Therefore, the central relationship for comparing measurements to theory is

$$
L_{3}=n_{l} C \frac{\hbar}{m} a^{4}
$$

In the recombination process the molecular binding en$\operatorname{ergy} \varepsilon$ is set free as kinetic energy. The molecule and the third atom receive $\varepsilon / 3$ and $2 \varepsilon / 3$, respectively. Since usually $\varepsilon$ is large compared to the trapping potential depth, both are expelled from the trap, setting $n_{l}=3$. However, the binding energy of the weakly bound last energy level of the dimer is given by $\varepsilon=\hbar^{2} /\left(m a^{2}\right)$ [15], and at very large scattering length values $\varepsilon$ may be below the trap depth and the third atom cannot escape. If the potential of the atom trap does not confine the molecule, the dimer is lost and $n_{l}=2$. If, however, the molecule is trapped and stays within the atom cloud, it may quickly quench its high vibrational excitation in an inelastic collision with a fourth trapped atom. The large amount of energy released in this situation expels the collision partners, making $n_{l}=3$. In either case, the kinetic energy of the remaining atom is distributed in the ensemble, giving rise to recombination heating.

Our measurements are conducted in a nearly isotropic optical dipole trap formed by two crossed $100 \mathrm{~W} \mathrm{CO}_{2}$ laser beams focused to a waist of $600 \mu \mathrm{m}$ [16]. A vertical magnetic field gradient of $31 \mathrm{G} / \mathrm{cm}$ cancels gravity for 
the $6^{2} \mathrm{~S}_{1 / 2}, F=3, m_{F}=3$ absolute ground state of Cs. The optical trapping forces are so weak that no other $m_{F}$ substate can be held against the gravitational force, thus perfect spin polarization of the sample is enforced by the trap itself. We have measured the trap frequencies by exciting small oscillations along the principal axes, the geometric average results to $\bar{\omega} /(2 \pi)=14.5 \pm 0.5 \mathrm{~Hz}$. The trap depth is $k_{B} \times 12 \mu \mathrm{K} ; k_{B}$ denotes the Boltzmann constant. Trap heating is extremely weak, from longtime measurements we can give an upper bound for the heating rate of $0.2 \mathrm{nK} / \mathrm{s}$. The background gas limited $1 / e$ lifetime is $\sim 180 \mathrm{~s}$.

After loading the dipole trap [16], the ensemble cools down by plain evaporation within the first $10 \mathrm{~s}$ to $3 \times 10^{6}$ atoms at $T=1 \mu \mathrm{K}, 1 / 12$ of the trap depth. To avoid further evaporative loss and cooling in our measurements, we cool the sample well below this limit. This is achieved by forced radio frequency evaporative cooling in the magnetic levitation field. We apply a radio frequency resonant with the Zeeman splitting between two $m_{F}$ substates. In the vertical levitation gradient this acts as height selective removal from the trap, effecting 1D evaporative cooling. Evaporation proceeds at a bias field of $B=75 \mathrm{G}$ to tune the scattering length to $1200 a_{0}$ ( $a_{0}=0.053 \mathrm{~nm}$ denotes Bohr's radius), which at the low initial peak density of $3 \times 10^{11} \mathrm{~cm}^{-3}$ keeps the elastic scattering rate at $\sim 200 \mathrm{~s}^{-1}$, high enough for efficient cooling. A simple $7 \mathrm{~s}$ two-segment linear sweep of the radio frequency provides $1.3 \times 10^{6}$ atoms at $450 \mathrm{nK}$ (peak phasespace density $D \approx 4 \times 10^{-3}$ ). Some measurements have been taken at $200 \mathrm{nK}$ starting temperature; to reach this, we appended another linear $5 \mathrm{~s}$ rf ramp, leaving $4 \times 10^{5}$ atoms in the trap $\left(D \approx 10^{-2}\right)$. Temperature and atom number are measured by taking an absorption image with typically $50 \mathrm{~ms}$ of expansion in the levitation field after switching off the $\mathrm{CO}_{2}$ lasers. For the expansion, the bias field is in all measurements set to $75 \mathrm{G}$ to ensure constant conditions.

In a first set of measurements, we have recorded the atom number and temperature of the trapped sample after a holding time of $10 \mathrm{~s}$ at fixed magnetic fields varied between $10 \mathrm{G}$ and $100 \mathrm{G}$ [17]. Figure 1] shows the data in comparison to the calculated scattering length [14], which includes Feshbach resonances involving $s$ - and $d$ wave channels. Additional resonances involving closed $g$-wave channels are marked by arrows. The Feshbach resonances have been observed in previous experiments through light-induced two-body losses [13, 18] and described theoretically [12, 18]. In our measurements, all resonances appear as prominent loss and heating features. Over the full range, both atom number and temperature show a clear correlation with the magnitude of the scattering length.

To obtain more quantitative results on recombination loss and heating, we have studied the time evolution of the atom number $N$ and temperature $T$ at various mag-

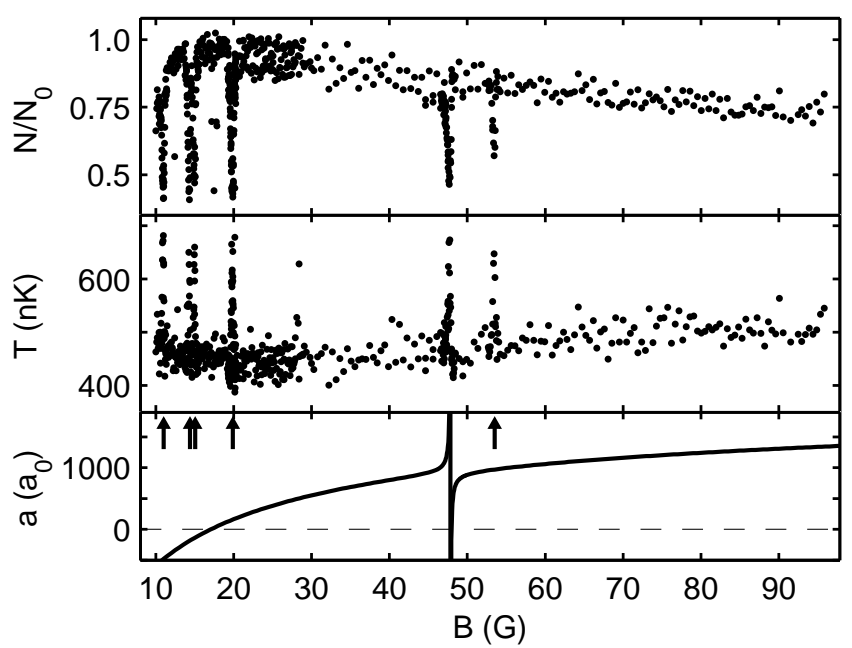

FIG. 1: Remaining fraction of atoms $N / N_{0}$ (upper curve) and temperature $T$ (middle) after $10 \mathrm{~s}$ in the $\mathrm{CO}_{2}$-laser trap in a range of magnetic bias fields. Initially $N_{0}=1.3 \times 10^{6}$ atoms are prepared at $450 \mathrm{nK}$. For comparison, the lower plot shows the calculated scattering length $a$ with additional Feshbach resonances indicated by arrows (see text).

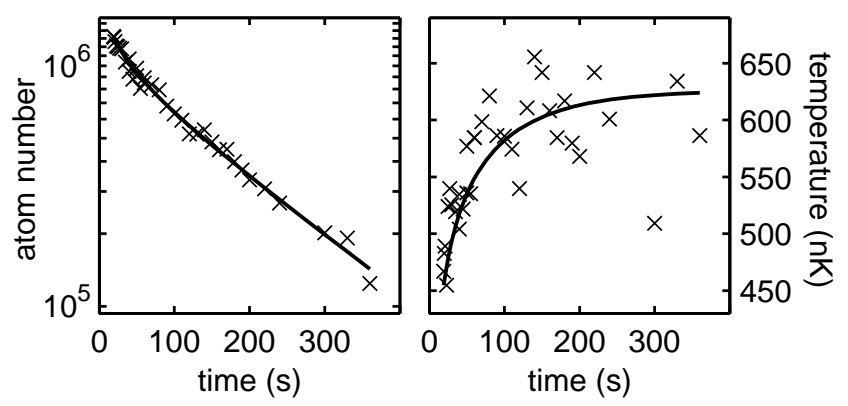

FIG. 2: Atom number and temperature as a function of time in the trap at a bias field of $56 \mathrm{G}$. The solid lines show a numerical fit to the data. The corresponding three-body loss coefficient is $L_{3}=7(2) \times 10^{-25} \mathrm{~cm}^{6} / \mathrm{s}$.

netic fields. Figure 2 shows a typical measurement at $B=56 \mathrm{G}$. Integrating the local three-body loss rate $L_{3} n^{3}$ over the sample gives $\dot{N} / N=-L_{3}\left\langle n^{2}\right\rangle$. Expressing the average density in terms of the directly accessible quantities $N$ and $T$, we replace $L_{3}\left\langle n^{2}\right\rangle$ by $\gamma N^{2} / T^{3}$, where $\gamma=L_{3}\left(m \bar{\omega}^{2} / 2 \pi k_{B}\right)^{3} / \sqrt{27}$. With an additional loss term $-\alpha N$ for background gas collisions, we obtain a differential equation for the atom number,

$$
\frac{d N}{d t}=-\alpha N-\gamma \frac{N^{3}}{T^{3}} .
$$

Atom loss through three-body recombination leads to "anti-evaporation" heating. With its $n^{3}$ dependence, three-body recombination predominantly happens in the region of highest density in the trap center. The mean potential energy [19] of an atom undergoing a threebody process can be calculated by integrating over a 


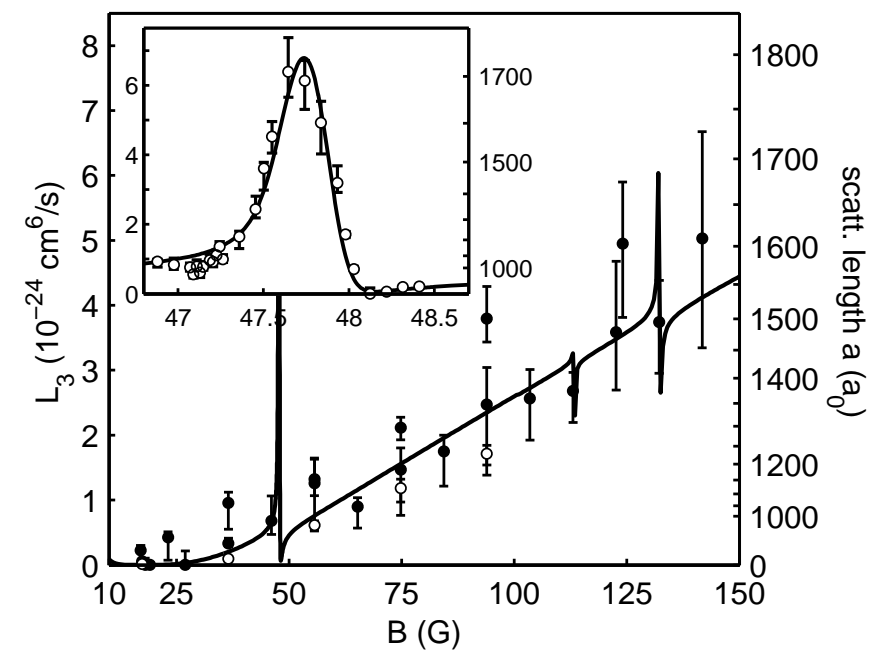

FIG. 3: Measured values of $L_{3}$ as a function of the bias field $B$. Filled symbols represent measurements taken at an initial temperature of $200 \mathrm{nK}$, open symbols at $450 \mathrm{nK}$. The solid line shows the calculation according to Eq. (1) with $n_{l} C=225$. The scale on the right hand side displays the corresponding scattering length values. The inset shows the region around the $48 \mathrm{G}$ resonance with a model fit (see text).

thermal distribution with weights proportional to $n^{3}$, which yields $\frac{1}{2} k_{B} T$. As the ensemble average is $\frac{3}{2} k_{B} T$, for each lost atom an excess energy of $1 k_{B} T$ remains in the sample. To account for recombination heating, we introduce an additional constant energy $k_{B} T_{h}$ per lost atom. Relating the total heating energy $k_{B}\left(T+T_{h}\right)$ to the average energy $3 k_{B} T$ of a trapped particle yields $\dot{T} / T=(\dot{N} / N) k_{B}\left(T+T_{h}\right) /\left(3 k_{B} T\right)$, and we obtain an expression for the temperature evolution,

$$
\frac{d T}{d t}=\gamma \frac{N^{2}}{T^{3}} \frac{\left(T+T_{h}\right)}{3} .
$$

Equations (2) and (3) form a set of two coupled nonlinear differential equations for $N$ and $T$. Using an iterative approach [20], we do a least-squares fit of the numerical solutions of both equations to the experimental data. For the data in Fig. 2 the fit is plotted as solid lines. Fit parameters are $\alpha, \gamma$ and $T_{h}$. Error estimates for the fit parameters are based on varying one parameter up and down, resp., to the points where $\chi^{2}$ increases by one, while optimizing the other parameters [22].

The data obtained for $L_{3}$ as a function of magnetic field is plotted in Fig. 3 The inset shows an expanded view of data taken on the $48 \mathrm{G}$ Feshbach resonance. The error bars represent the statistical errors derived from the fit as described above, which typically range around $20 \%$. The systematic uncertainty is larger: Due to the scaling of $L_{3}$ with $N^{2} \bar{\omega}^{6}$, small errors in these values amount to large differences in the result obtained for $L_{3}$. Taking the uncertainty in the trap frequency and an estimated $30 \%$ in atom number, the systematic error can be up to a factor of two.

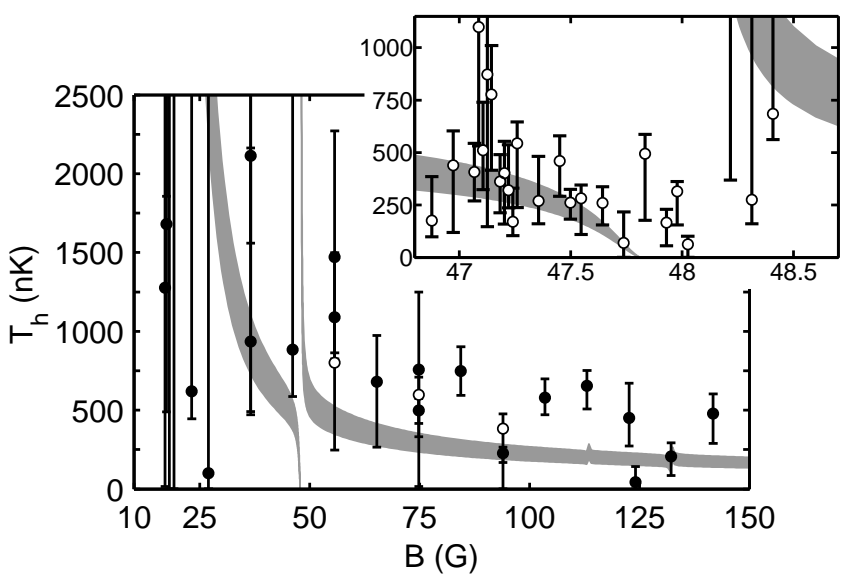

FIG. 4: Recombination heat $T_{h}$ as a function of the bias field $B$. Filled symbols represent measurements taken at an initial temperature of $200 \mathrm{nK}$, open symbols at $450 \mathrm{nK}$. The inset shows the region around the $48 \mathrm{G}$ resonance. The shaded area shows the expected range based on the calculated binding energy of the last bound state of the $\mathrm{Cs}_{2}$ molecule (see text).

In order to compare our data to theory, we do a leastsquares fit of the expected scaling law [Eq. (10] to our measurements, using the calculated $a(B)$ [14]. Single fit parameter is $n_{l} C$. The resulting curve is drawn as a solid line in Fig. 3. The data clearly confirm the universal $a^{4}$ scaling. In our experimental range, where $a$ varies by less than a factor of two, we do not find any indication of resonant behavior in $C$ [23]. The result from the fit is $n_{l} C=225$, corresponding to $C=75$ for $n_{l}=3$ or $C=112$ for $n_{l}=2$. In comparison to theory, both values are somewhat above the upper limit of $C_{\max } \approx 70$, but in good agreement within our systematic error limit.

The inset in Fig. 3 shows the data on the 48 G Feshbach resonance. In order to model the shape of the loss feature, we have to take into account the width of the sample in the vertical $B$ field gradient and the unitarity limitation at the finite temperature. The theory curve is convoluted with a gaussian of full $1 / e$ width $260 \mathrm{mG}$, which is given by the vertical extension of the $n^{3}$ distribution of the trapped sample. A cutoff in $a$ accounts for the unitarity limitation; the cutoff value of $1800 a_{0}$ results from a least-squares fit to the data. Our $B$-field scale is slightly adjusted for an optimum match with theory by introducing a $40 \mathrm{mG}$ shift, which is well within our calibration accuracy.

Via the condition $k a \ll 1$, the inverse wavenumber $k^{-1}=\left[4 k_{B} T m /\left(\pi \hbar^{2}\right)\right]^{-1 / 2}$ characterizes the crossover to the zero-energy limit. At $450 \mathrm{nK}, k^{-1}=1500 a_{0}$; at $200 \mathrm{nK}, k^{-1}=2250 a_{0}$. Correspondingly, the $450 \mathrm{nK}$ data on the Feshbach resonance show a strong effect from the unitarity limitation, while the data taken at $200 \mathrm{nK}$ starting conditions follow the expected scaling law up to the maximum $a \approx 1500 a_{0}$.

Our results on recombination heating are displayed in 
Fig. 4 A trend towards smaller $T_{h}$ at higher magnetic fields, corresponding to higher scattering lengths $a$, is visible. At large values of $a$ close to the Feshbach resonance, the values are correspondingly very small. We compare the data to the scenario that the recombination heating arises just from the binding energy $\varepsilon$ of the last bound state of the dimer. As the atom involved in the threebody recombination process receives $2 \varepsilon / 3$, the recombination heat per lost atom results to $k_{B} T_{h}=2 \varepsilon /\left(3 n_{l}\right)$. Therefore, we assume $k_{B} T_{h}$ to be between $2 \varepsilon / 9\left(n_{l}=3\right)$ and $\varepsilon / 3\left(n_{l}=2\right)$. This range is shown as shaded area in Fig. 4 [24]. This simple model shows reasonable agreement with the experimental data. Our heating measurements thus provide evidence of the formation of very weakly bound ultracold molecules.

From the apparent effect of recombination heating, we can draw an important conclusion for the efficiency of evaporative cooling towards quantum degeneracy in gases with large positive scattering length. If temperatures are very low $\left(k_{B} T \ll \varepsilon\right)$ and the atom that carries $2 \varepsilon / 3$ cannot immediately escape from the trap, recombination heating has a detrimental effect much worse than mere three-body loss. For efficient evaporation it is thus imperative to avoid the hydrodynamic collision regime and to implement a three-dimensional evaporation scheme. In our cooling experiments on optically trapped cesium [16] these conditions indeed turned out to be essential for the attainment of Bose-Einstein condensation.

The molecules formed in recombination are likely to remain trapped in our setup. A previous experiment shows evidence of trapping of the $\mathrm{Cs}_{2}$ dimer in a $\mathrm{CO}_{2}$-laser trap 26]. Away from Feshbach resonances, the magnetic dipole moment of the dimer in its last bound state has the same value as the sum of the dipole moments of two single Cs atoms, so that the levitation trap can hold the molecules under the same conditions as the atoms. This offers an intriguing way to study atom-molecule interactions both in ultracold thermal clouds and Bose-Einstein condensates. Moreover, the Feshbach resonances allow to change the molecular magnetic moment in a controlled way and thus to separate the molecules from the atoms to prepare pure molecular samples.

We thank C. Greene for useful discussions and P. Julienne for providing us with the scattering length data. We gratefully acknowledge support by the Austrian Science Fund (FWF) within SFB 15 (project part 16) and by the European Union in the frame of the Cold Molecules TMR Network under contract No. HPRN-CT-2002-00290.

[1] F. Masnou-Seeuws and P. Pillet, Adv. At. Mol. Opt. Phys. 47, 53 (2001).

[2] E. A. Donley, N. R. Claussen, S. T. Thompson, and C. E.
Wieman, Nature 417, 529 (2002).

[3] J. Söding, D. Guéry-Odelin, P. Desbiolles, F. Chevy, H. Inamori, and J. Dalibard, Appl. Phys. B 69, 257 (1999).

[4] E. Burt, R. Ghrist, C. Myatt, M. Holland, E. Cornell, and C. Wieman, Phys. Rev. Lett. 79, 337 (1997).

[5] D. M. Stamper-Kurn, M. R. Andrews, A. P. Chikkatur, S. Inouye, H.-J. Miesner, J. Stenger, and W. Ketterle, Phys. Rev. Lett. 80, 2027 (1998).

[6] J. Stenger, S. Inouye, M. R. Andrews, H.-J. Miesner, D. M. Stamper-Kurn, and W. Ketterle, Phys. Rev. Lett. 82, 2422 (1999).

[7] J. L. Roberts, N. R. Claussen, S. L. Cornish, and C. E. Wieman, Phys. Rev. Lett. 85, 728 (2000).

[8] P. O. Fedichev, M. W. Reynolds, and G. V. Shlyapnikov, Phys. Rev. Lett. 77, 2921 (1996).

[9] E. Nielsen and J. H. Macek, Phys. Rev. Lett. 83, 1566 (1999).

[10] B. D. Esry, C. H. Greene, and J. P. Burke, Jr., Phys. Rev. Lett. 83, 1751 (1999).

[11] P. F. Bedaque, E. Braaten, and H.-W. Hammer, Phys. Rev. Lett. 85, 908 (2000).

[12] P. J. Leo, C. J. Williams, and P. S. Julienne, Phys. Rev. Lett. 85, 2721 (2000).

[13] C. Chin, V. Vuletic, A. J. Kerman, and S. Chu, Phys. Rev. Lett. 85, 2717 (2000).

[14] Calculations by P. S. Julienne, E. Tiesinga, and C. J. Williams (2003), using the model of [12]. Private communication.

[15] L. D. Landau and E. M. Lifshitz, Quantum Mechanics: Non-Relativistic Theory (Pergamon Press, Oxford, 1977), 3rd ed.

[16] T. Weber, J. Herbig, M. Mark, H.-C. Nägerl, and R. Grimm, Science 299, 232 (2003), published online 5 Dec 2002 (10.1126/science.1079699).

[17] Below $10 \mathrm{G}$, the levitation gradient distorts the trapping potential too strongly for consistent measurements [16].

[18] C. Chin, Ph.D. thesis, Stanford University (2001).

[19] In the low-temperature limit, the recombination process does not depend on the kinetic energy.

[20] Iteration steps are as follows: I. Choose values for $\alpha$ and $\gamma$. II. Using these values, calculate a best fit of the solution of Eq. (3) to the temperature measurements, with $T_{h}$ as single fit parameter. III. Calculate the error sum of squares $e_{N}$ between the atom number measurements and the solution of Eq. (2). Steps I-III are repeated while varying $\alpha$ and $\gamma$ to minimize $e_{N}$ using a nonlinear optimization algorithm [21].

[21] MATLAB 6.5 R13, optimization toolbox, function lsqnonlin.

[22] P. R. Bevington and D. K. Robinson, Data reduction and error analysis for the physical sciences (McGrawHill, New York, 1992), 2nd ed.

[23] $C$ is predicted to undergo one full oscillation period between 0 and $C_{\max }$ when $a$ varies by a factor of 22.711 .

[24] We include a correction 25] giving $\varepsilon=\hbar^{2} /\left(m(a-\bar{a})^{2}\right)$, with $\bar{a}=95.5 a_{0}$ for cesium.

[25] G. F. Gribakin and V. V. Flambaum, Phys. Rev. A 48, 546 (1993).

[26] T. Takekoshi, B. M. Patterson, and R. J. Knize, Phys. Rev. Lett. 81, 5105 (1998). 